\title{
p73 regulates DRAM-independent autophagy that does not contribute to programmed cell death
}

\author{
D Crighton ${ }^{1}$, J O'Prey ${ }^{1}$, HS Bell ${ }^{1,2}$ and KM Ryan ${ }^{\star, 1}$
}

Evading programmed cell death is a common event in tumour development. The $p 53$ family member, $p 73$, is a potent inducer of death and a determinant of chemotherapeutic response, but different to $p 53$, is rarely mutated in cancer. Understanding cell death pathways downstream of $\mathrm{p} 53$ and p73 is therefore pivotal to understand both the development and treatment of malignant disease. Recently, p53 has been shown to modulate autophagy - a membrane trafficking process, which degrades long-lived proteins and organelles. This requires a p53 target gene, DRAM, and both DRAM and autophagy are critical for p53-mediated death. We report here that TA-p73 also regulates DRAM and autophagy, with different TA-p73 isoforms regulating DRAM and autophagy to varying extents. RNAi knockdown of DRAM, however, revealed that p73's modulation of autophagy is DRAMindependent. Also, p73's ability to induce death, again different to $\mathrm{p53}$, is neither dependent on DRAM nor autophagy. In contrast to TA-p73, $\Delta \mathrm{N}-\mathrm{p} 73$ is a negative regulator of p53-induced and p73-induced autophagy, but does not affect autophagy induced by amino-acid starvation. These studies, therefore, represent not only the first report that p73 modulates autophagy but also highlight important differences in the mechanism by which starvation, p53 and p73 regulate autophagy and how this contributes to programmed cell death.

Cell Death and Differentiation (2007) 14, 1071-1079. doi:10.1038/sj.cdd.4402108; published online 16 February 2007

In higher eukaryotes, programmed cell death pathways represent a major protective mechanism against malignant disease. ${ }^{1}$ These pathways are stimulated when cells experience adverse conditions to prevent the propagation of mutated or damaged cells that may otherwise go on to form a tumour. As a result, inactivation of cell death pathways is a common event in many types of cancer. ${ }^{2}$ Many standard forms of chemotherapy also utilize programmed cell death pathways to invoke a therapeutic response. ${ }^{3}$ As a result, the inactivation of these pathways during tumour development poses a serious problem for clinical success. However, the mechanism by which cell death is regulated during tumour development and in cancer therapy remains unclear. The further investigation, therefore, of the factors and pathways controlling tumour cell death should lead not only to a better understanding of the aetiology of cancer but also to improved strategies and novel targets for therapeutic intervention.

One of the major factors controlling tumour cell death is the tumour suppressor p53. ${ }^{4}$ The importance of cell death to tumour suppression is exemplified by $\mathrm{p} 53$, which is mutated in $50 \%$ of all human cancers. ${ }^{5}$ In response to various forms of cellular stress, including DNA damage, hypoxia and oncogene activation, p53 levels become elevated. ${ }^{4}$ After a small proportion of p53 localizes to mitochondria - to stimulate cell death by interaction with members of the Bcl-2 family ${ }^{6-8}-$ the majority of p53 accumulates in the nucleus where it activates a number of target genes. ${ }^{1,4}$ These genes regulate a spectrum of biological processes, for example growth arrest and DNA repair, but an ever increasing number invoke cell death through a variety of mechanisms to ultimately stimulate the apoptotic cascade. . $^{1,4,9,10}$

p53 has recently also been linked to another cellular process that controls cell death - autophagy. ${ }^{11,12}$ Autophagy (strictly the form of autophagy known as macroautophagy, but hereafter for simplicity referred to as autophagy) is a vesicular trafficking process that mediates the degradation of long-lived proteins and is the only pathway within the cell for the degradation of organelles. ${ }^{13}$ The basal levels of autophagy in a cell can vary from cell type to cell type, but can be stimulated in response to various forms of stress. Upon induction, a membrane structure known as the 'isolation membrane' is formed de novo from an unknown origin. This structure grows to form a double-membraned vesicle containing bulk cytoplasm and organelles - the autophagosome. These autophagosomes then mature, perhaps fusing with endosomes, before fusing with lysosomes. The acidic hydrolases of the lysosome then effect degradation of the contents of the autophagosome, resulting in amino acids and fatty acids that can then be either metabolized for energy production or recycled into biosynthetic pathways. ${ }^{14}$

Autophagy has been reported to have both roles in cell death and cell survival. ${ }^{15}$ In response to nutrient deprivation, for example, the induction of autophagy can act as a selflimited survival mechanism, by degrading bulk cytoplasm and

\footnotetext{
${ }^{1}$ Tumour Cell Death Laboratory, Beatson Institute for Cancer Research, Cancer Research UK Beatson Laboratories, Garscube Estate, Glasgow, UK ${ }^{*}$ Corresponding author: KM Ryan, Tumour Cell Death Laboratory, Beatson Institute for Cancer Research, Cancer Research UK Beatson Laboratories, Garscube Estate, Switchback Rd, Glasgow G61 1BD, UK. Tel: + 44141330 3655; Fax: + 44141942 6521; E-mail: k.ryan@ @eatson.gla.ac.uk

${ }^{2}$ Current address: Department of Biomedical Science, University of Aberdeen, UK

Keywords: p53; p73; autophagy; apoptosis; DRAM

Abbreviations: TetOn, tetracycline-responsive; TA-, transactivation-competent; Dox, doxycycline; qPCR, quantitative-PCR; Tam, tamoxifen; CHX, cycloheximide; Met/ Cys, methionine/cysteine; DRAM, damage-regulated autophagy modulator; mTOR, mammalian target of rapamycin

Received 22.9.06; revised 15.12.06; accepted 04.1.07; Edited by G Melino; published online 16.2.07
} 
organelles for energy production until a sufficient supply of exogenous nutrients is restored. ${ }^{16-18}$ In other situations, however, autophagy has been reported to be a pro-death mechanism either by inducing type II 'autophagic cell death' or by linking with other cell death pathways, including apoptosis, to effect the demise of the cell. ${ }^{19,20}$ In tumour development, therefore, autophagy can be considered, depending on the context, to act in either an oncogenic or tumour suppressive capacity. p53 has been reported to be an inducer of autophagy. ${ }^{11,12}$ Morever, the recent discovery that DRAM a p53 target gene that is required for p53-induced autophagy is frequently down-regulated in squamous cancers underscores the notion that autophagy is a component of tumour suppression downstream of p53. ${ }^{12}$

p73 is a protein that is closely related to $p 53$. $^{21,22}$ It shares many functional similarities with p53, for example, both have been reported to mediate cell cycle arrest and programmed cell death. ${ }^{23}$ Clearly though, the two proteins also have divergent functions. This is perhaps most exemplified in studies of mouse development in which p53 appears largely dispensable, whereas p73-null animals exhibit clear developmental abnormalities. ${ }^{24,25}$ In addition, during tumour development, in contrast to $\mathrm{p} 53, \mathrm{p} 73$ is rarely mutated, indicating different contributions of the two proteins to tumour suppres- sion. ${ }^{26}$ Several studies have shown that that these differences may lie in the spectrum of target genes activated by p53 and p73 in specific contexts and how these integrate to determine a cell's fate. ${ }^{27}$ We sought therefore to determine whether $\mathrm{p} 73$, like p53, can regulate DRAM and autophagy and whether this contributes to p73-mediated programmed cell death.

\section{Results}

p73 is an inducer of autophagy. To investigate if p73 modulates autophagy, we utilized previously characterized tetracycline-responsive (TetOn) cell lines for three of the transactivation-competent (TA) isoforms of $\mathrm{p} 73$ : TA-p73 $\alpha$, TA-p73 $\beta$ and TA-p73 $\gamma{ }^{28}$ Upon treatment of these cells with the tetracycline analogue, doxycycline (Dox) each line undergoes a marked induction in expression of the transgene (Figure 1a). Before induction each of the lines, as well as a p53 inducible line (TetOn-p53), were infected with an adenovirus expressing the autophagy marker GFP. LC3. ${ }^{29}$ In most unstimulated cells, endogenous LC3 exists in a form LC3I, which shows a diffuse distribution within the cytoplasm. When autophagy is induced, LC3I is lipidated to form LC3II which integrates into the autophagosome
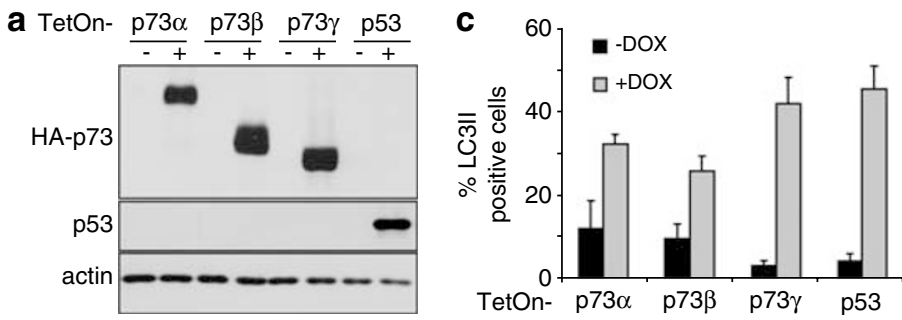

b

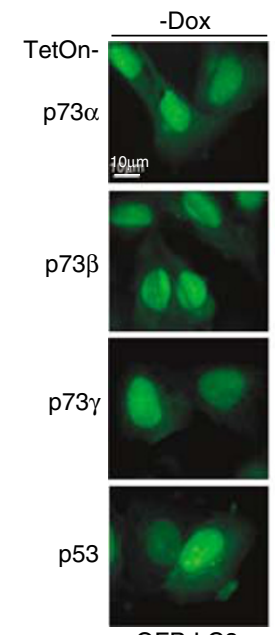

GFP-LC3

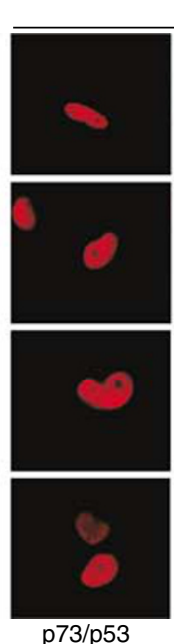

+ Dox

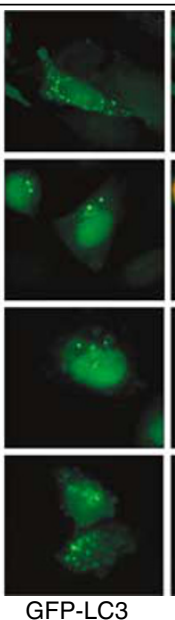

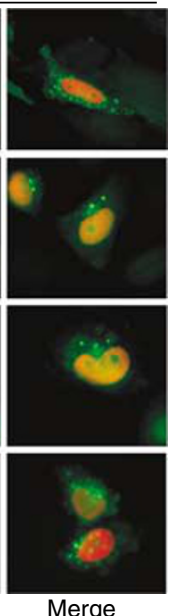

d

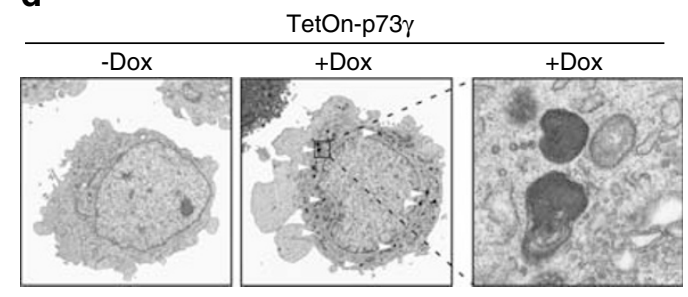

e

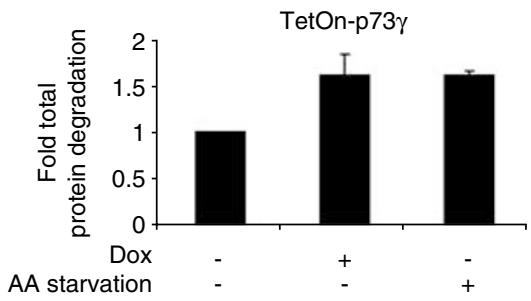

$\mathbf{f}$

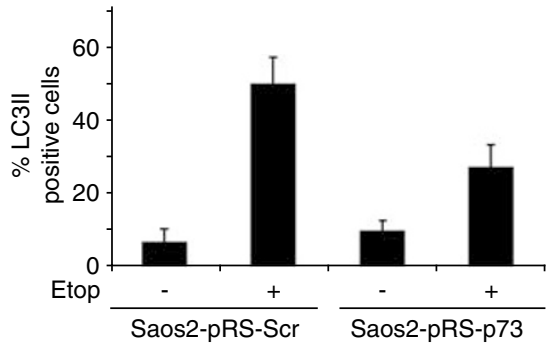

Figure 1 p73 regulates autophagy. (a-c) TetOn-p73 $\alpha,-p 73 \beta,-p 73 \gamma$ and -p53 cells were infected with an adenovirus expressing GFP-LC3 then induced for $24 \mathrm{~h}$ with Dox. Cells were then assessed for transgene induction by Western blotting (a) and visualized for the appearance of GFP-LC3Il puncta (b). GFP-LC3 is shown in green and HAp73 $\alpha,-$ p73 $\beta,-p 73 \gamma$ and -p53 are shown in red. (c) Cells were quantified for the appearance of GFP-LC3 puncta. Cells positive for p73 $\alpha$, p73 $\beta$, p73 $\gamma$ or p53 were counted. (d) The presence of autophagosomes in Dox-treated TetOn-p73 $\gamma$ cells was confirmed by transmission electron microscopy (white arrowheads highlight a selection of autophagosomes) (e) and the ability of p73 to induct successful autophagy was measured by analysis of long-lived protein degradation. (f) The involvement of p73 in the induction of autophagy following treatment with etoposide (Etop) was assessed in Saos2 cells containing an shRNA to knockdown p73 (pRS-p73) or a scrambled non-silencing shRNA control (pRS-Scr) 
membrane. ${ }^{30}$ When this occurs, the exogenous marker GFPLC3 behaves similarly and can be easily observed as distinct puncta under fluorescent microscopy. ${ }^{29}$ Although only a few GFP-LC3 puncta could be observed in each of the lines in the absence of Dox, a large number of puncta were clearly visible following induction of each of the splice variants of p73 (Figure 1b). No increase in puncta was observed when parental Saos2 cells were treated with Dox (Supplementary Figure 1). TA-p73 $\gamma$ had the strongest effect, with approximately $50 \%$ of transgene-positive cells having a significant number of GFP-LC3 puncta (above 8 per cell) a score similar to what is observed following p53 induction (Figure 1c). TA-p73 $\alpha$ and TA-p73 $\beta$ had a less potent effect on autophagy with approximately $20-30 \%$ of the p73-postive cells showing significant levels of GFP-LC3 puncta - despite similar levels of transgene expression to TA-p73 $\gamma$ following treatment with Dox (Figure 1a and c).

Next, we analysed cells for the appearance of autophagosomes by electron microscopy (EM). Morphologically, autophagosomes are double-membraned vesicles that contain cytoplasm and/or organelles. These are most obvious in the later stages of autophagy when the autophagosomes contain at least partially degraded material and appear more electron dense. ${ }^{14}$ Consistent with our findings with GFP-LC3, treatment of TetOn-p73 $\gamma$ cells with Dox caused a marked induction of electron dense vesicles (Figure 1d, middle panel). When these vesicles were examined under higher magnification, they were clearly double-membraned and contained cellular material (Figure 1d, right panel).

The autophagosome is a transient mid-point in the autophagy process. As such, accumulation of autophagasomes in cells can either indicate induction of autophagy or a blockade in the turnover of autophagosomes. As autophagy is the major route by which long-lived proteins are degraded in cells, we monitored the degradation rate of these proteins in the absence or presence of $p 73$. TetOn-p73 $\gamma$ cells were radio-labelled with $\left[{ }^{35} \mathrm{~S}\right] \mathrm{Met} / \mathrm{Cys}$ and treated with Dox or cultured in the absence of amino acids (a known inducer of autophagy). ${ }^{13}$ Starvation and p73 expression both caused comparable increases in the degradation of long-lived proteins, indicating that p73 is an inducer of autophagy (Figure 1e).

Lastly, we sought to determine whether endogenous p73 could modulate autophagy. To do this, we utilized Saos 2 cells, which are null for p53 but have been shown to induce p73 following treatment with the chemotherapeutic drug etoposide. ${ }^{31}$ To determine the contribution of endogenous p73, these cells were infected with either a scrambled shRNA or a previously described shRNA designed to knockdown p73. ${ }^{32}$ In line with our findings with exogenous $p 73$, the subsequent infection of these cells with GFP-LC3 and treatment with etoposide revealed a marked induction in the appearance of autophagosomes that was greatly reduced in cells expressing the shRNA targeting p73 (Figure 1f).

p73 induces DRAM, but this is not required for p73mediated autophagosome formation. Our previous studies have indicated that p53-induced autophagy requires induction of the recently identified p53 target gene, DRAM. ${ }^{12}$ We therefore tested if DRAM was also a target gene of $\mathrm{p} 73$.
Following induction of the p73 isoforms and p53 for $24 \mathrm{~h}$, RNA was isolated and the levels of DRAM determined by quantitative RT-PCR (qPCR). This revealed that although TA-p73 $\gamma$ was a strong inducer of DRAM expression (fourfold), TA-p73 $\alpha$ and $-\beta$ induced only small, but reproducible increases in DRAM mRNA (Figure 2a). Each of the lines, however, was able to activate significant levels of p21 (Figure 2b). This therefore indicates that the low level of $D R A M$ activation by TA-p73 $\alpha$ and $-\beta$ is genuine and not due to a lack of functional competence for these splice variants.

To determine if the effect of p73 on DRAM was direct, we generated a $\mathrm{H} 1299$ cell line (a p53-null cell line from a carcinoma of the lung), which contains a p73-ER fusion protein. This switchable system can be used to modulate the activity of $p 73$ by addition of the oestrogen analogue, tamoxifen (Tam). ${ }^{33}$ Activation of p73-ER with Tam does not require protein synthesis and as a result changes in mRNA expression, which occur in the presence of a protein synthesis inhibitor, such as cycloheximide ( $\mathrm{CHX})$, can be considered direct. In this system, DRAM was markedly induced following treatment with Tam either in the absence or presence of $\mathrm{CHX}$ (Figure 2c) demonstrating a direct effect of p73 on DRAM expression.

To further examine the direct nature of the ability of p73 to activate DRAM, we examined if $p 73$ could activate a luciferase reporter construct containing a fragment of DRAM intron 1, which has previously been shown to be p53 responsive. ${ }^{12}$ Consistent with our observations of DRAM activation in TetOn-p73 $\gamma$ cells (Figure 2a), co-transfection of TA-p73 $\gamma$ with this reporter caused an approximately sixfold increase in luciferase activity (Figure 2d). However, also consistent with our analysis of activation of endogenous DRAM (Figure 2a), this was considerably lower than the 12-fold induction observed following transfection of p53 (Figure 2d).

As the ability of the p73 isoforms to induce autophagosome accumulation correlated with the ability to induce DRAM, we next asked if $D R A M$ induction was required for p73 to modulate autophagy. Initially, we focused our analysis on TA-p73 $\gamma$ owing to its pronounced effects on autophagy and DRAM. TetOn-p73 $\gamma$ cells were transfected with three DRAMspecific siRNAs and a non-targeting siRNA control. Each of these DRAM siRNAs caused a marked reduction in both basal $D R A M$ and the levels of DRAM seen following treatment with Dox (Figure 3a). To test the effects of DRAM knockdown on autophagy in whole populations of p73-expressing cells, we utilized the fact that, as well as being detectable as puncta by immunofluorescence, LC3II migrates faster than LC3I on SDS-PAGE gels. ${ }^{29,30}$ TetOn-p73 $\gamma$ cells were transfected with $D R A M$-specific and non-targeting siRNAs and subsequently infected with GFP-LC3. Cell lysates were analysed $48 \mathrm{~h}$ later by Western blotting to determine the ratio of GFP-LC3I/GFPLC3II (Figure 3b). For comparison, we assayed one of the DRAM-specific siRNAs for its ability to knockdown DRAM and to affect the ratio of LC3I/LC3II in TetOn-p53 cells (Figure 3c and d). Taken together, these data revealed that, in contrast to what was observed in TetOn-p53 cells where a clear reduction in GFP-LC3II was observed (Figure 3d), knockdown of DRAM had no affect on the GFP-LC3I/GFP-LC3II ratio in TetOn-p73 $\gamma$ cells (Figure $3 b$ ). This indicates that p73-mediated modulation of autophagy is DRAM independent. 


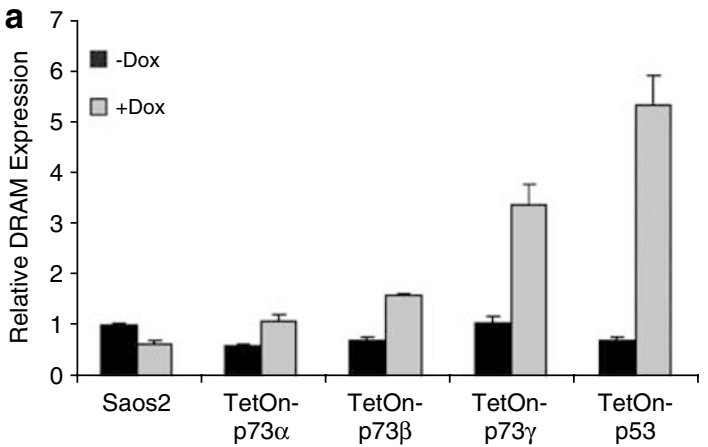

C

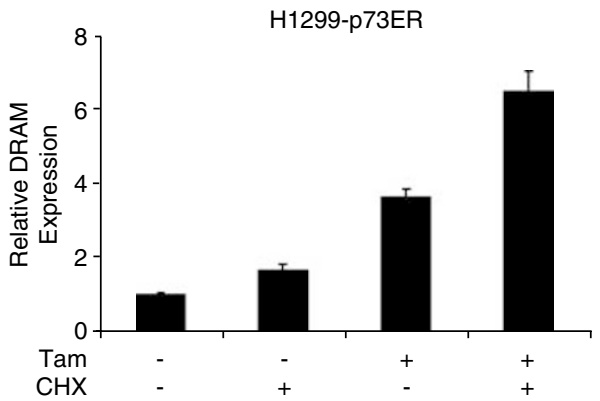

b

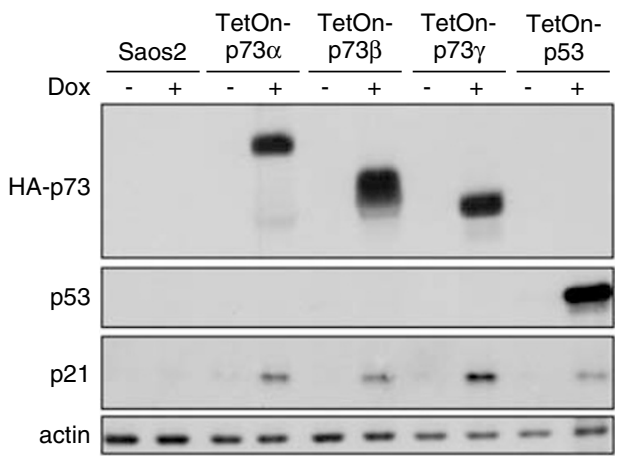

d

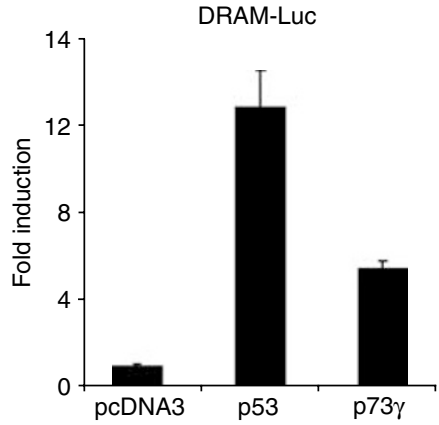

Figure 2 p73 induces DRAM. (a) TetOn-p73 $\alpha,-p 73 \beta,-p 73 \gamma$ and $-p 53$ cells were induced with Dox for $24 \mathrm{~h}$, RNA was extracted and the relative amounts of DRAM mRNA was assessed by quantitative RT-PCR. (b) Protein levels of p $73 \alpha, \mathrm{p} 73 \beta, \mathrm{p} 73 \gamma$, p53 and p21 were analysed by Western blotting. The ability of p73 to directly activate DRAM was assessed in H1299-p73-ER cells (c) and in a reporter assay with a luciferase construct-containing sequences from DRAM intron 1 (d)

p73-mediated induction of DRAM is not required for programmed cell death. Our previous studies had also observed that as well as being required for $\mathrm{p} 53$ to induce autophagy, DRAM was also required for p53-mediated programmed cell death. ${ }^{12}$ We first therefore questioned if the ability of $\mathrm{p} 73$ isoforms to induce programmed cell death correlated with expression of DRAM. TetOn-p73 $\alpha,-\beta$ and $-\gamma$ cells were treated with Dox for $48 \mathrm{~h}$ and the extent of resulting apoptosis measured by flow cytometry. Different to what we observed for activation of DRAM by the p73 isoforms, where activation was most pronounced following induction by TA$\mathrm{p} 73 \gamma$, we found that levels of cell death were comparable following activation of TA-p73 $\beta$ and $-\gamma$ (Figure 4a). In contrast, only a small amount of apoptosis was seen following activation of $p 73 \alpha$ (Figure $4 a$ ).

We next sought to determine therefore whether DRAM was contributing to the cell death observed. TetOn-TA-p73 $\gamma$ cells were transfected with the three DRAM-specific siRNAs and a non-targeting siRNA control as outlined in Figure $3 a$. For comparison, analysis was also made of the effects of DRAM knockdown in TetOn-p53 cells (Figure 4b). Cells were then incubated in either the absence or presence of Dox and the extent of cell death determined by flow cytometry. None of the DRAM-specific siRNAs caused any significant effect on the cell death seen following activation of TA-p73 $\gamma$ (Figure 4c); however, consistent with our previous studies, DRAM knockdown caused a reduction in the amount of cell death observed following activation of p53 (Figure 4d).
Induction of autophagy by p73 does not contribute to programmed cell death. Our data indicate that in contrast to p53, when DRAM is induced by p73, it does not appear to play a role in either p73's modulation of autophagy or programmed cell death. This however does not rule-out the possibility that p73-induced autophagy is contributing to cell death in a DRAMindependent manner. To test this, we transfected TetOn-TAp73 $\gamma$ and TetOn-p53 cells with a previously described siRNA targeting the essential autophagy gene $A T G 5^{12}$ and a nontargeting control. Following ATG5 silencing, which was confirmed by qPCR (Figure $5 \mathrm{a}$ and b), the ability of p53 and $\mathrm{p} 73 \gamma$ to modulate autophagy, as determined by analysis of LC3I/LC3II ratios by Western blotting, was markedly reduced (Figure $5 \mathrm{c}$ and d). We next analysed if ATG5 knockdown in these cells affected programmed cell death. Consistent with our previous studies, ATG5 knockdown caused a statistically significant $(P=0.008)$ decrease in amount of cell death seen from p53 when compared with cells transfected with nontargeting siRNA control (Figure $5 \mathrm{c}$ ). ${ }^{12}$ In contrast, no reduction in cell death was observed following ATG5 knockdown in TAp73 $\gamma$ cells (Figure $5 d$ ). A small but reproducible increase in death was observed, however, this was not found to be statistically significant $(P>0.05)$. These data clearly indicate that, in contrast to p53, the modulation of autophagy by TAp73 $\gamma$ does not contribute to programmed cell death.

$\Delta \mathrm{N}-\mathrm{p} 73$ modulates p53- and p73- driven autophagy, but does not affect starvation-driven autophagy. p73 splice variants also exist in forms with an alternate $\mathrm{N}$-terminus, 
a

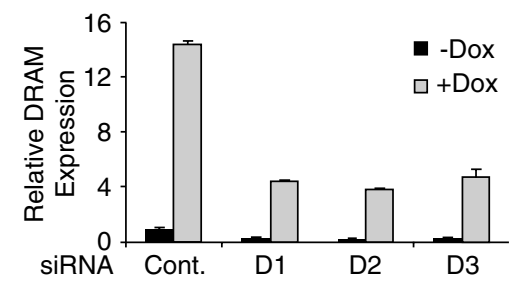

b
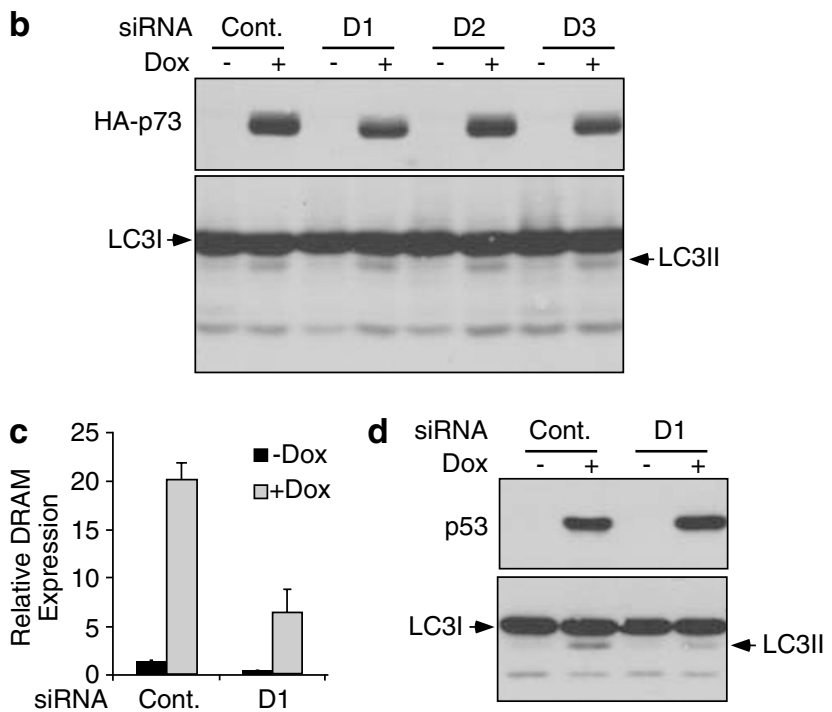

Figure 3 Silencing of DRAM does not affect p73-induced autophagy. Control or DRAM-specific siRNAs were transfected into TetOn-p73 $\gamma$ (a and $\mathbf{b}$ ) or TetOn-p53 (c and d) cells for a total of $72 \mathrm{~h}$. The relative levels of DRAM were assessed by realtime PCR after $24 \mathrm{~h}$ of induction with Dox (a and $\mathbf{c}$ ). Following transfection, the cells were infected with an adenovirus expressing GFP-LC3 and induced with Dox for $48 \mathrm{~h}$. Levels of GFP-LC31/II and either p73 $\gamma$ (b) or p53 (d) were analysed by Western blotting. Cont., non-targeting control siRNA. D1, D2 and D3, DRAMspecific siRNAs

termed collectively $\Delta \mathrm{N}-\mathrm{p} 73 .^{26}$ These splice variants lack the ability to transactivate genes, but as they bind the same DNA consensus sequences as TA-p73 and p53, they can act as transdominant-negative regulators of transactivation. As the ability of p73 to induce autophagy appears DRAMindependent and therefore possibly transactivation independent, we asked whether $\Delta \mathrm{N}-\mathrm{p} 73$ could modulate autophagy per se and whether $\Delta \mathrm{N}-\mathrm{p} 73$ could modulate the induction of autophagy in a negative manner. To test this, Saos2 cells were infected with an adenovirus expressing $\Delta \mathrm{N}$ p73 and GFP-LC3. Unlike TA-p73 (Figure 1) or amino-acid starvation, $\Delta \mathrm{N}-\mathrm{p} 73$ does not cause accumulation of autophagosomes (Figure 6a). However, $\Delta \mathrm{N}$-p73 was able to repress the formation of autophagosomes induced by $p 53$ or TA-p73 (Figure $6 b-e$ ), indicating that although p73-driven autophagy may not be dependent on DRAM, it is likely to be dependent on other target gene(s) of TA-p73. In contrast $\Delta \mathrm{N}$ p73 had no effect on starvation-induced autophagy. This implies three independent autophagy signalling pathways: p53-driven that is DRAM-dependent, p73-driven that is DRAM-independent and starvation induced that is independent of both p53 and TA-p73. p53 does not contribute to p73-induced autophagy. For induction of cell death in certain systems, cooperativity between p53 family members has been shown to be important. We considered therefore whether the ability of p73 to induce autophagy in cell types containing wild-type p53, may be at least partially dependent on p53. We therefore analysed the ability of TA-p73 to induce autophagy in U2OS cells, which like Saos2 cells are from an osteosarcoma, but unlike Saos2, contain wild-type p53. Similar to what we observed in Saos2 cells (Figure 1), infection of U2OS cells with an adenovirus expressing TAp73 caused a marked increase in GFP-LC3 puncta (Figure $7 \mathrm{a}$ ). To test if this effect was in any way dependent on p53, we infected these cells with a shRNA to knockdown $\mathrm{p} 53^{34}$ (Figure $7 \mathrm{~b}$ ). These cells and cells infected with a nonsilencing shRNA as control were then infected with adenoviral TA-p73 and GFP-LC3. This revealed however that knockdown of p53 had no effect on the ability of p73 to induce autophagy (Figure 7c).

\section{Discussion}

To our knowledge, this is the first report which shows that p73 can modulate autophagy. It therefore introduces yet another regulator of this process that has also been shown to control cell viability. Our previous studies and those of others have reported that $\mathrm{p} 53$ can modulate autophagy. ${ }^{11,12}$ It is perhaps, therefore, no surprise that its closely related family member, p73, can also do so. It is intriguing, however, to find that the mechanism by which autophagy is regulated by $p 73$ is different to that of p53. p53's regulation of autophagy is critically dependent on the activation of its target gene, DRAM. ${ }^{12}$ Although DRAM is directly activated by $p 73$, it appears that it is dispensable for p73 to modulate autophagy. p53 has also been shown to modulate autophagy by signalling through the nutrient-sensing kinase, mTOR. ${ }^{11}$ Whether this signal is upstream or downstream of DRAM, or for that matter completely independent is as yet unknown. It would be intriguing to assess if p73 can affect mTOR activity and whether this is involved in the regulation of autophagy by $p 73$. It is also entirely possible that another p73 target gene, distinct from DRAM, may mediate p73-induced autophagy and our studies with $\Delta \mathrm{N}-\mathrm{p} 73$ would indicate that this is likely. The identification of such a target gene(s) and an assessment of whether this is also a target of p53 would undoubtedly be very rewarding.

We also report that the regulation of autophagy by $p 73$ does not contribute to $p 73$-mediated programmed cell death. This is clearly an important functional difference between p53 and p73 and it is tempting to speculate that this may underlie critical fundamental differences between the two proteins in, for example, tumour suppression. What then is the role of autophagy downstream of p73? Clearly, we cannot completely rule-out the possibility that autophagy induced by p 73 may contribute to cell death in some situations. For example, it is possible that under nutrient-deprived conditions, where autophagy is a critical regulator of cell viability, that the autophagy induced by p73 may be important. This would be an intriguing possibility regardless of whether autophagy under these conditions promotes cell death or cell survival. 
a

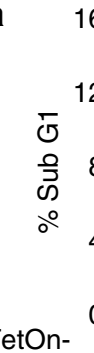

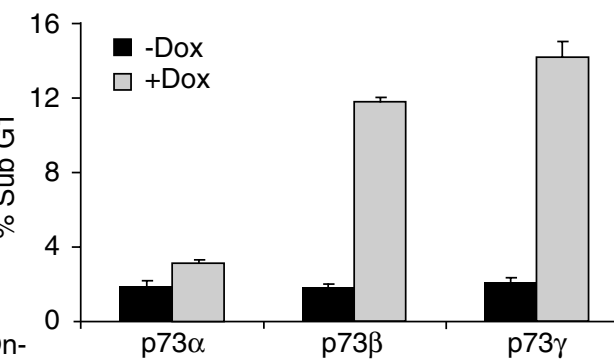

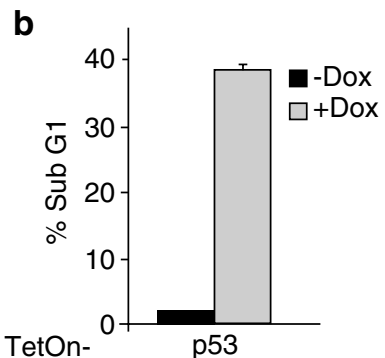

C

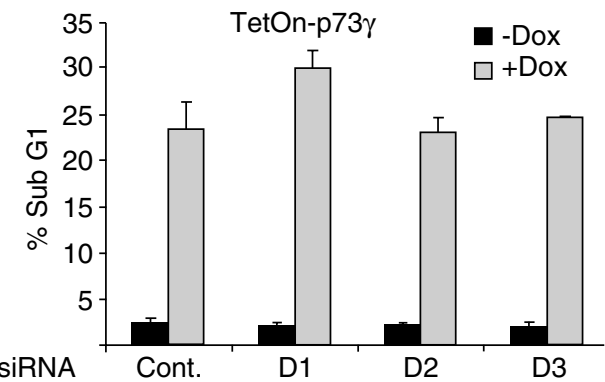

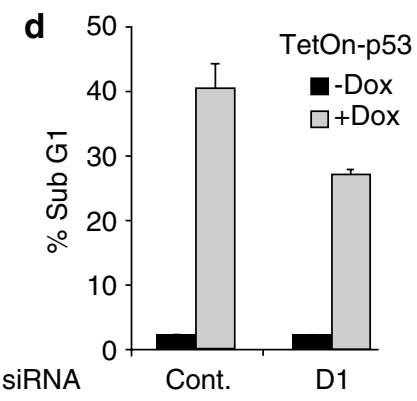

Figure 4 Induction of DRAM is not required for p73-mediated programmed cell death. (a-b) p73- $\beta$ and p73- $\gamma$ induce programmed cell death. TetOn-p73 $\alpha$, p73 $\beta$, p73 $\gamma$ cells (a) and p53 cells (b) were induced with Dox for $48 \mathrm{~h}$ and the amount of apoptosis determined by flow cytometry. Apoptosis was measured by the $\%$ sub-G1 content of the cells. (c-d) Knockdown of DRAM affects death from p53, but not p73. Contol or DRAM-specific siRNAs were transfected into TetOn-p73 $(\mathbf{c}$ ) or TetOn-p53 (d) cells for a total of $72 \mathrm{~h}$. Cells were induced with Dox for $48 \mathrm{~h}$ and the amount of apoptosis was determined by flow cytometry. Apoptosis was measured by the \% sub-G1 content of the cells. Cont., non-targeting control siRNA. D1, D2 and D3, DRAM-specific siRNAs

a

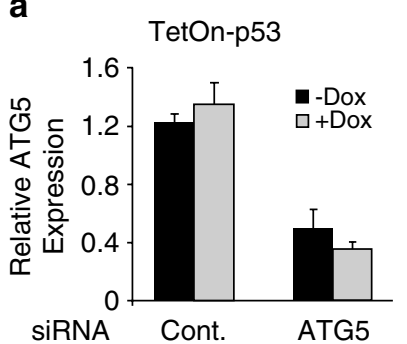

b

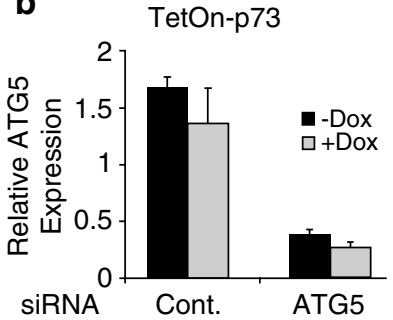

C

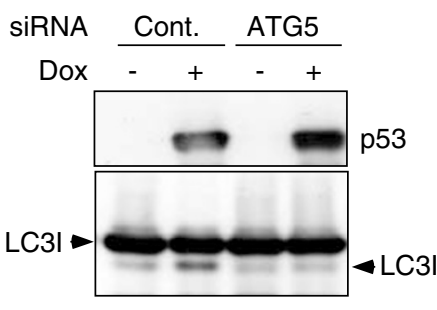

TetOn-p53

d

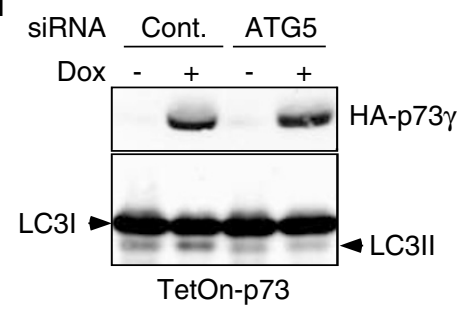

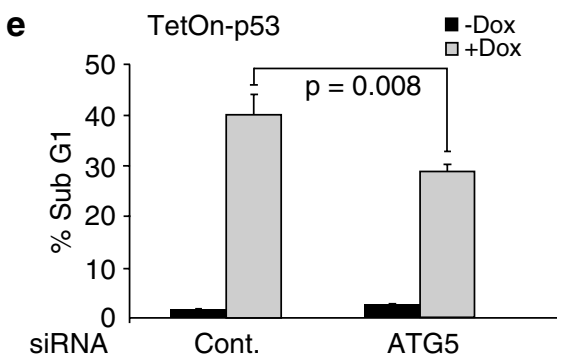

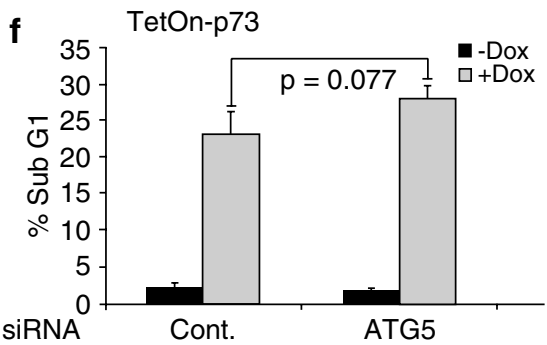

Figure 5 Autophagy is not required for p73-induced apoptosis. TetOn-p53 (a, c, e) and TetOn-TA-p73 $\gamma$ (b, d, f) cells were transfected with control or ATG5-specific siRNAs for a total of $72 \mathrm{~h}$. The relative levels of ATG5 were assessed by real-time PCR after $24 \mathrm{~h}$ induction with Dox $(\mathbf{a}$ and $\mathbf{b})$. The effects on autophagy were assessed after $48 \mathrm{~h}$ induction with Dox by measuring the ratio of GFP-LC3I to GFP-LC3Il by Western blotting (c and $\mathbf{d}$ ) and the effects on apoptosis determined by flow cytometry (e, f). Apoptosis was measured by the \% sub-G1 content of the cells. Cont., non-targeting control siRNA

Aside from a role in controlling cell viability during tumour development or starvation conditions, it may also be possible that $\mathrm{p} 73$ regulates autophagy to serve a completely different purpose in a very specific context. One notable difference between p53 and p73 is the role of p73 in development. ${ }^{24,25}$ Autophagy has been shown to have roles in cell death regulation and also tissue remodelling during development and it may well be that it is during development that p73's effects on autophagy play a role.

Our studies also raise questions about DRAM's role downstream of $p 73$. DRAM induction appears to play no role in the regulation of autophagy or programmed cell death by 

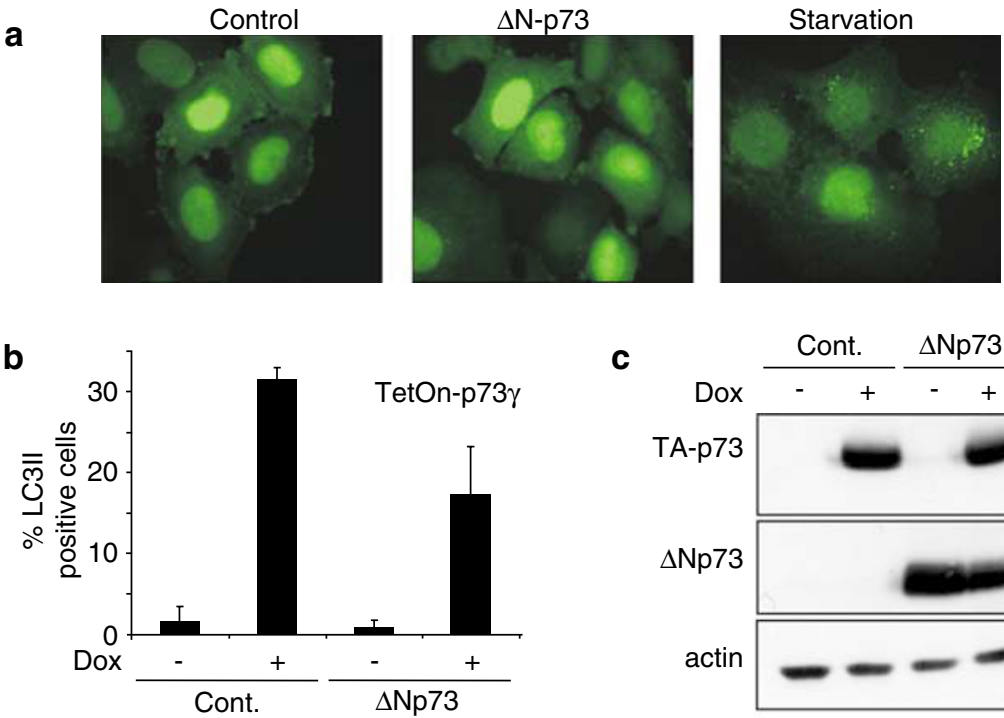

C
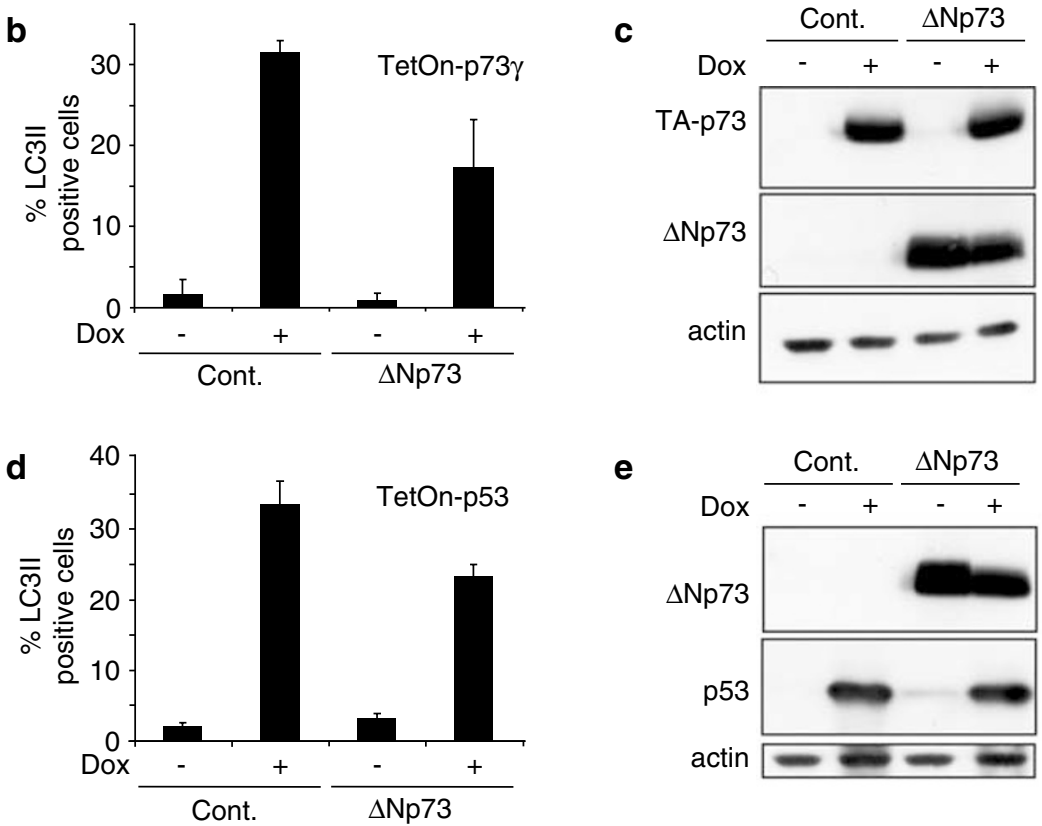

e
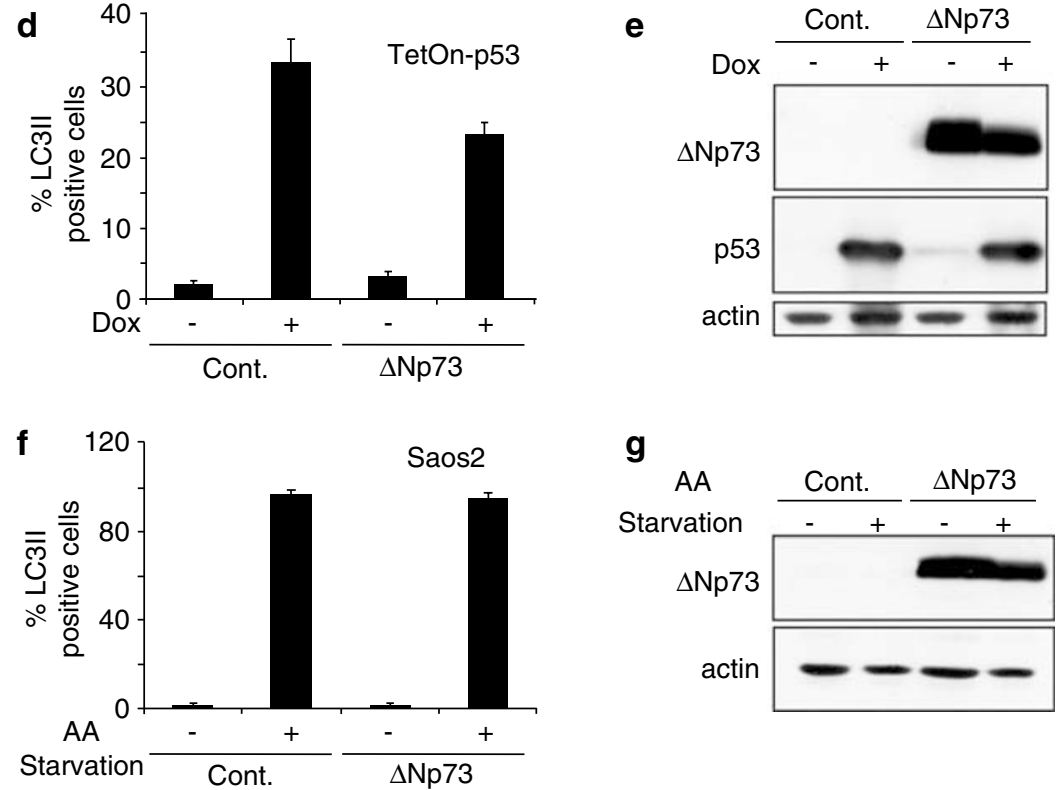

Figure $6 \Delta \mathrm{N}$-p73 regulates p53- and p73-driven autophagy, but does induce autophagy itself and does not inhibit starvation-driven autophagy. (a) $\Delta \mathrm{N}-\mathrm{p} 73 \mathrm{cells}$ do not exhibit accumulation of autophagosomes following infection with GFP-LC3. (b-g) Expression of $\Delta \mathrm{N}-\mathrm{p} 73$ impedes the ability of p53 and p73 to induce accumulation of autophagosomes, but does not affect starvation-induced autophagy. Accumulation of GFP-LC3 puncta was assayed by microscopy and quantified (b, d, f) and transgene expression was determined by Western blotting $(\mathbf{c}, \mathbf{e}, \mathbf{g})$. Cont., control 'empty' adenovirus

p73 under the conditions tested. As with the role of autophagy downstream of $p 73$, it may well be that DRAM contributes to p73 function, perhaps, even to p73-mediated programmed cell death, in a specific context. It may be for example, as DRAM is only strongly activated by $\mathrm{p} 73 \gamma$, that DRAM has specific functions in cells that express relatively large amounts of this p73 isoform. Equally, it still remains possible that although knockdown of DRAM does not affect p73's ability to modulate autophagy, it may be that the basal, or knockdown, levels of DRAM contribute to autophagy regulation in combination with other p73-induced genes. The generation of knockout cells/mice would clearly help resolve this issue.
Ultimately, however, the function of DRAM per se requires further investigation before these issues can really be addressed. Although the specific function of DRAM is unknown, its lysosomal localization would indicate likely roles in either trafficking through the secretory pathway or in the degradation of proteins that are delivered to the lysosome. It is possible that specific cellular stresses that target these processes elicit a p73-dependent response where DRAM is critical. It is clear, therefore, that further studies are required to elucidate what role DRAM and indeed autophagy play downstream of $p 73$ and how this relates to the differences in function between $\mathrm{p} 53$ and p73. 

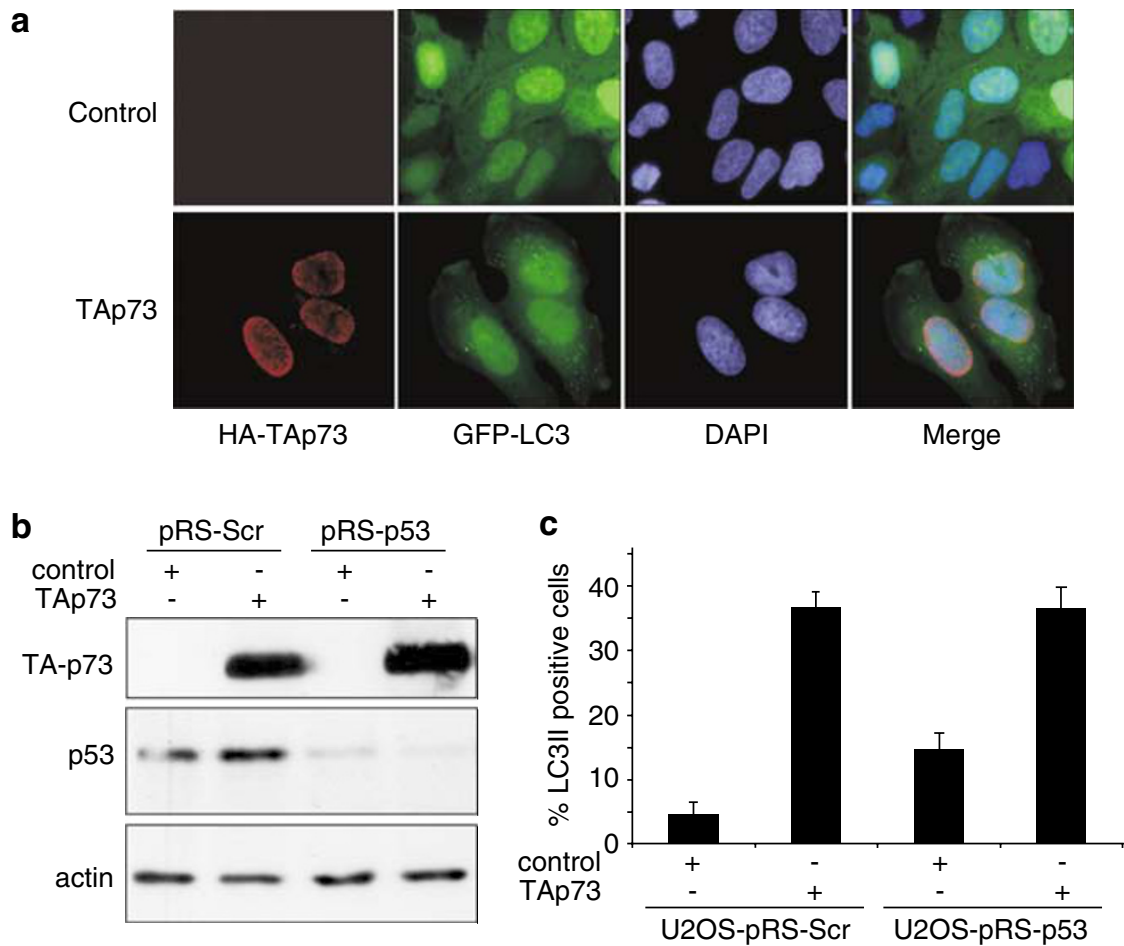

Figure 7 p53 is not a component of p73-driven autophagy in wild-type p53 expressing cells. (a) Infection of U2OS cells with adenoviral TA-p73 causes accumulation of GFP-LC3 punta. (b, c) p73-induced autophagy is not affected by knockdown of p53 by RNAi. Expression of p53 and p73 was determined by Western blotting (b). Accumulation of GFP-LC3 puncta was visualized by fluorescent microscopy and quantified (c). Control, control 'empty' adenovirus

\begin{abstract}
Materials and Methods
Plasmids. pShuttleCMV-HA-TAp73 $\alpha$ and pShuttleCMV-HA- $\triangle N p 73 \alpha$ were created by cloning HA-TAp73 $\alpha$ and HA- $\triangle$ Np $73 \alpha$ fragments from pcDNA3-HA vectors (kindly donated by Gerry Melino) into pShuttleCMV (kindly provided by Bert Vogelstein $)^{35}$ using $\mathrm{Kpnl}$ and Xhol restriction enzymes. PretroSUPER-Scr, pRetroSUPER-p53 and pRetroSUPER-p73, ${ }^{12,32}$ pcDNA3-p53, pcDNA3-HATAp73 $\gamma$ and DRAM-Luc (BS-1 Luc) have been described previously. ${ }^{12,36}$ pBabePuro-p73-ER was generated by fusing coding sequences of p73 to the hormone-binding domain of the oestrogen receptor as described previously. ${ }^{33}$ The p73-ER fusion was then subsequently cloned into $\mathrm{pBabePuro}$.
\end{abstract}

Cell culture and transfetions. Saos2, U2OS and H1299 cells can all be obtained from ATCC. TetOn-p53, TetOn-73 $\alpha$, TetOn-p73 $\beta$ and TetOnp73 $\gamma$ cells are Saos2-derived lines and have been described previously. ${ }^{28,37}$ Saos2-pRS-Scr and Saos2-pRS-p73 were generated by infection with pRetroSUPER-Scr (pRS-Scr) and pRetroSUPER-p73 (pRS-p73). U2OS-pRS-Scr and U2OS-pRS-p53 were generated by infection of U2OS cells with pRetroSUPER-Scr (pRS-Scr) and pRetroSUPER-p53 (pRS-p53), respectively. H1299-p73-ER cells were generated

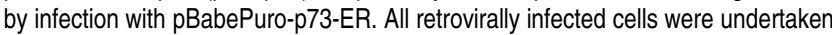
as previously described ${ }^{38}$ and all infected cells were selected with $1 \mu \mathrm{g} / \mathrm{ml}$ puromycin (Sigma). All cell lines were maintained in Dulbecco's modified Eagle's medium supplemented with $10 \%$ foetal bovine serum. Induction of transgene expression was achieved by addition of $1 \mu \mathrm{g} / \mathrm{ml}$ doxyclycline (Dox) (Sigma). For starvation conditions, cells were incubated in Earle's Balanced Salt Solution (EBSS) (Sigma). Where indicated, cells were transfected by $\mathrm{CaPO}_{4}$ precipitation as previously described. ${ }^{39}$

Western blotting. Cells were lysed in a $2 \times$ Western sample buffer and transferred to nitrocellulose membranes as described previously. ${ }^{39}$ Membranes were probed using standard immunoblotting techniques with antibodies that recognize p73 (HRP-conjugated HA, Roche), p53 (DO-1, Pharmingen), p21 (sc397G, Santa Cruz) and actin (clone 1A4, Sigma).
Generation of adenoviruses. Linearized 'Shuttle' plasmids were electroporated into BJ5183-AD-1 electro-competent cells (Stratagene) containing the Adeasy-1 adenoviral construct. Recombinant plasmids were then amplified in XL10-Gold cells (Stratagene) and transfected into HEK293 cells after restriction digest with Pacl. Following amplification in 293 cells, purified adenovirus was isolated by freeze-thaw extraction and titred using the BD Bioscience Adeno- $X$ Rapid Titer kit. Adenoviruses were added to cultures at a concentration of 12 infectious units per cell.

Long-lived protein degradation assays. TetOn-p 73 cells were labelled for $6 \mathrm{~h}$ with L- $\left[{ }^{35} \mathrm{~S}\right] \mathrm{Met} / \mathrm{Cys}$ ( $5 \mu \mathrm{Ci} / \mathrm{ml}$ ) (Amersham), washed three times in PBS and incubated for a further $16 \mathrm{~h}$ in DMEM supplemented with $2 \mathrm{mM}$ unlabelled L-Met/Cys plus Dox where indicated. The degradation period was started by washing the cells again and replacing with fresh medium or EBSS. After $4 \mathrm{~h}$, the levels of degraded protein were calculated as previously described. ${ }^{18}$

Cell death assays. Total populations of cells, including floating and adherent cells, were processed for flow cytometric analysis (FACScan, Becton Dickinson) as described previously. ${ }^{40}$ Acquired events were analysed with CellQuest software (Becton Dickinson). The percentage of cells with a sub-G1 DNA content was taken as a measure of the extent of apoptosis in the cell population at that time.

Luciferase reporter assays. Saos2 cells were transfected with $5 \mu \mathrm{g}$ of DRAM-Luc, and $0.5 \mu \mathrm{g}$ of pcDNA3-p53, pcDNA-p73 or empty vector. $24 \mathrm{~h}$ later, cells were lysed in luciferase lysis buffer (Promega) according to the manufacturer's instructions. Samples were assayed for luciferase activity.

Immunofluoresence. For analysis of autophagosomes, cells were infected with an adenovirus expressing GFP-LC3 (a kind gift from Aviva Tolkovsky, University of Cambridge). ${ }^{29}$ Sixteen hours later, cells were incubated either in the absence of presence of Dox. After a further $24 \mathrm{~h}$, cells were fixed with $4 \%$ paraformaldehyde and permeabilized with $0.3 \%$ triton. Cells were then stained with antibodies that recognize exogenous p73 (HA - clone 12CA5, Roche) or p53 (DO-1, 
Pharmingen). Following incubation with a Texas red-conjugated secondary antibody (Molecular Probes Inc.), cells were analysed by confocal microsopy.

Electron microscopy. Following treatments, cells were fixed with $2 \%$ glutaraldehyde in $0.1 \mathrm{M}$ sodium cacodylate and transmission electron microscopy performed using standard techniques.

Quantitative RT-PCR. RNA was prepared using TRIzol Reagent (Invitrogen) qPCR analysis was undertaken using the DyNAmo SYBR Green 2-step qRT-PCR kit (Finnzymes). Data collection was carried out using a Chromo4 real-time PCR detector and analysed with Opticon Monitor 3. Primers for DRAM and 18S have been described previously. ${ }^{12}$ Primers for ATG5 were: (forward) GCCTGTATGTACTGCTTTAACTC, (reverse) GGATAATGCCATTTCAGTGGT qPCR cycling parameters were $95^{\circ} \mathrm{C} 15 \mathrm{~min}\left[94^{\circ} \mathrm{C} 10 \mathrm{~s}, 55^{\circ} \mathrm{C} 30 \mathrm{~s}, 72^{\circ} \mathrm{C} 30 \mathrm{~s}\right.$ ] 34 cycles, $72^{\circ} \mathrm{C} 10 \mathrm{~min}$. Expression levels of genes analysed by qPCR were normalized relative to levels of $18 \mathrm{~S}$ rRNA.

RNAi. Desalted and deprotected synthetic oligonucleotides were purchased from Dharmacon. The siRNA sequences targeting DRAM were: AGCCACGATG TATACAAGA (1), CCACAGAAATCAATGGTGA (2) and GGACAGTGGCCTTT GGTTT (3). The sequence for ATG5 was CATCTGAGCTACCCGGATA and the non-silencing sequence was TAAGGCTATGAAGAGATAC. siRNAs were transfected using oligofectamine reagent (Invitrogen).

Statistical analyses. For statistical comparison of the effects of ATG knockdown on cell death in TetOn-p53 and TetOn-p73 cells, the Student's t-test was applied assuming equal variances. Using a Levine test, there was no evidence to suggest that variances were unequal (Supplementary Table 1).

Acknowledgements. We thank Reuven Agami, Bert Vogelstein, Karen Vousden, Aviva Tolkovsky, Gerry Melino, Kanaga Sabapathy, Trevor Littlewood and Noboru Mizushima for reagents and Gareth Inman, Dan Croft, Simon Wilkinson and Laura Bell for critical reading of the paper. We are also extremely grateful to Ken Blight and Lucy Collinson for electron microscopy and to Jacqui Hall for statistics. Work in the Tumour Cell Death Laboratory is supported by Cancer Research UK. $\mathrm{KR}$ is a Cancer Research UK Senior Cancer Research Fellow.

1. Crighton D, Ryan KM. Splicing DNA-damage responses to tumour cell death. Biochim Biophys Acta 2004; 1705: 3-15.

2. Hanahan D, Weinberg RA. The hallmarks of cancer. Cell 2000; 100: 57-70.

3. Johnstone RW, Ruefli AA, Lowe SW. Apoptosis: a link between cancer genetics and chemotherapy. Cell 2002; 108: 153-164.

4. Ryan KM, Phillips AC, Vousden KH. Regulation and function of the p53 tumor suppresso protein. Curr Opin Cell Biol 2001; 13: 332-337.

5. Soussi T, Lozano G. p53 mutation heterogeneity in cancer. Biochem Biophys Res Commun 2005; 331: 834-842.

6. Mihara M, Erster S, Zaika A, Petrenko O, Chittenden T, Pancoska $P$ et al. p53 has a direct apoptogenic role at the mitochondria. Mol Cell 2003; 11: 577-590.

7. Leu JI, Dumont $\mathrm{P}$, Hafey M, Murphy ME, George DL. Mitochondrial p53 activates Bak and causes disruption of a Bak-Mcl1 complex. Nat Cell Biol 2004; 6: 443-450.

8. Chipuk JE, Kuwana T, Bouchier-Hayes L, Droin NM, Newmeyer DD, Schuler M et al. Direct activation of Bax by p53 mediates mitochondrial membrane permeabilization and apoptosis. Science 2004; 303: 1010-1014.

9. Laptenko 0 , Prives $C$. Transcriptional regulation by p53: one protein, many possibilities Cell Death Differ 2006; 13: 951-961.

10. Michalak E, Villunger A, Erlacher M, Strasser A. Death squads enlisted by the tumou suppressor p53. Biochem Biophys Res Commun 2005; 331: 786-798.

11. Feng $\mathrm{Z}$, Zhang $\mathrm{H}$, Levine AJ, Jin S. The coordinate regulation of the $\mathrm{p} 53$ and $\mathrm{mTOR}$ pathways in cells. Proc Natl Acad Sci USA 2005; 102: 8204-8209.
12. Crighton D, Wilkinson S, O'Prey J, Syed N, Smith P, Harrison PR et al. DRAM, a p53induced modulator of autophagy, is critical for apoptosis. Cell 2006; 126: 121-134

13. Klionsky DJ, Emr SD. Autophagy as a regulated pathway of cellular degradation. Science 2000; 290: 1717-1721.

14. Eskelinen EL. Maturation of autophagic vacuoles in mammalian cells. Autophagy 2005; 1 : $1-10$

15. Baehrecke EH. Autophagy: dual roles in life and death? Nat Rev Mol Cell Biol 2005; 6: 505-510.

16. Kuma A, Hatano M, Matsui M, Yamamoto A, Nakaya $\mathrm{H}$, Yoshimori T et al. The role of autophagy during the early neonatal starvation period. Nature 2004; 432: 1032-1036.

17. Lum JJ, Bauer DE, Kong M, Harris MH, Li C, Lindsten T et al. Growth factor regulation of autophagy and cell survival in the absence of apoptosis. Cell 2005; 120: 237-248.

18. Boya P, Gonzalez-Polo RA, Casares N, Perfettini JL, Dessen P, Larochette N et al. Inhibition of macroautophagy triggers apoptosis. Mol Cell Biol 2005; 25: 1025-1040.

19. Shimizu S, Kanaseki T, Mizushima N, Mizuta T, Arakawa-Kobayashi S, Thompson CB et al. Role of $\mathrm{Bcl}-2$ family proteins in a non-apoptotic programmed cell death dependent on autophagy genes. Nat Cell Biol 2004; 6: 1221-1228.

20. Yu L, Alva A, Su H, Dutt P, Freundt E, Welsh $S$ et al. Regulation of an ATG7-beclin 1 program of autophagic cell death by caspase-8. Science 2004; 304: 1500-1502.

21. Kaghad M, Bonnet $\mathrm{H}$, Yang A, Creancier L, Biscan JC, Valent A et al. Monoallelically expressed gene related to $p 53$ at $1 p 36$, a region frequently deleted in neuroblastoma and other human cancers. Cell 1997; 90: 809-819.

22. Jost CA, Marin MC, Kaelin Jr WG. p73 is a simian (correction of human) p53-related protein that can induce apoptosis. Nature 1997; 389: 191-194.

23. De Laurenzi V, Melino G. Evolution of functions within the p53/p63/p73 family. Ann NY Acad Sci 2000; 926: 90-100.

24. Donehower LA, Harvey M, Slagle BL, McArthur MJ, Montgomery Jr CA, Butel JS et al. Mice deficient for p53 are developmentally normal but susceptible to spontaneous tumours. Nature 1992; 356: 215-221.

25. Yang A, Walker N, Bronson R, Kaghad M, Oosterwegel M, Bonnin J et al. p73-deficient mice have neurological, pheromonal and inflammatory defects but lack spontaneous tumours. Nature 2000; 404: 99-103.

26. Melino G, De Laurenzi V, Vousden KH. p73: Friend or foe in tumorigenesis. Nat Rev Cancer 2002; 2: 605-615.

27. Harms K, Nozell S, Chen X. The common and distinct target genes of the p53 family transcription factors. Cell Mol Life Sci 2004; 61: 822-842.

28. Nakano K, Balint $\mathrm{E}$, Ashcroft $\mathrm{M}$, Vousden $\mathrm{KH}$. A ribonucleotide reductase gene is a transcriptional target of p53 and p73. Oncogene 2000; 19: 4283-4289.

29. Bampton ETW, Goemans CG, Niranjan D, Mizushima N, Tolkovsky AM. The dynamics of autophagy visualized in live cells. Autophagy 2005; 1: 23-36.

30. Kabeya Y, Mizushima N, Ueno T, Yamamoto A, Kirisako T, Noda T et al. LC3, a mammalian homologue of yeast Apg8p, is localized in autophagosome membranes after processing. EMBO J 2000; 19: 5720-5728.

31. Rossi M, De Laurenzi V, Munarriz E, Green DR, Liu YC, Vousden KH et al. The ubiquitinprotein ligase Itch regulates p73 stability. EMBO J 2005; 24: 836-848.

32. Toh WH, Kyo S, Sabapathy K. Relief of p53-mediated telomerase suppression by p73. J Biol Chem 2005; 280: 17329-17338.

33. Littlewood TD, Hancock DC, Danielian PS, Parker MG, Evan GI. A modified oestrogen receptor ligand-binding domain as an improved switch for the regulation of heterologous proteins. Nucleic Acids Res 1995; 23: 1686-1690.

34. Brummelkamp TR, Bernards R, Agami R. A system for stable expression of short interfering RNAs in mammalian cells. Science 2002; 296: 550-553.

35. He TC, Zhou S, da Costa LT, Yu J, Kinzler KW, Vogelstein B. A simplified system for generating recombinant adenoviruses. Proc Natl Acad Sci USA 1998; 95: 2509-2514.

36. De Laurenzi V, Costanzo A, Barcaroli D, Terrinoni A, Falco M, Annicchiarico-Petruzzelli M et al. Two new p73 splice variants, gamma and delta, with different transcriptional activity. J Exp Med 1998; 188: 1763-1768.

37. Ryan KM, Ernst MK, Rice NR, Vousden KH. Role of NF-kappaB in p53-mediated programmed cell death. Nature 2000; 404: 892-897.

38. Ryan KM, O'Prey J, Vousden KH. Loss of nuclear factor-kappaB is tumor promoting but does not substitute for loss of p53. Cancer Res 2004; 64: 4415-4418.

39. Bell LA, O'Prey J, Ryan KM. DNA-binding independent cell death from a minimal proapoptotic region of E2F-1. Oncogene 2006; 25: 5656-5663.

40. Ryan KM, Vousden KH. Characterization of structural p53 mutants which show selective defects in apoptosis but not cell cycle arrest. Mol Cell Biol 1998; 18: 3692-3698.

\section{Supplementary Information accompanies the paper on Cell Death and Differentiation website (http://www.nature.com/cdd)}

